\title{
Triple-Staple Technique Effectively Reduces Operating Time for Rectal Anastomosis
}

\author{
Marie Shella De Robles ${ }^{1}$, Christopher John Young ${ }^{1,2}$ \\ ${ }^{1}$ Department of Colorectal Surgery, Royal Prince Alfred Hospital, Sydney; ${ }^{2}$ Discipline of Surgery, The University of Sydney, Sydney, Australia
}

Purpose: Stapled anastomotic techniques to the distal rectum have gained widespread acceptance due to their procedural advantages. Various modifications in the stapling techniques have evolved since their inception. The triple-staple technique utilizing stapled closure of both the proximal colon and distal rectal stump provides a rapid and secure colorectal anastomosis. The aims of this study were to determine the safety and efficacy of the triple-staple technique and to compare the clinical outcomes with a historical control group for which the conventional double-staple technique had been performed.

Methods: One hundred consecutive patients operated on by a single surgeon were included in the study; 50 patients who underwent a double-staple (DSA) procedure and 50 patients undergoing triple-staple anastomosis (TSA).

Results: The most common indication for surgery in both groups was rectal cancer followed by diverticular disease and distal sigmoid cancer. There was no significant difference in number of patients requiring loop ileostomy formation in the groups (TSA, $56.0 \%$ vs. DSA, 68.0\%; $\mathrm{P}=0.621$ ). The mean operating time for the TSA group was significantly shorter compared to that of the DSA group (TSA, 242.8 minutes vs. DSA, 306.1 minutes; $\mathrm{P}=0.001$ ). There was no significant difference in complication rate (TSA, $40 \%$ vs. DSA, $50 \%$; $\mathrm{P}=0.315$ ) or length of hospital stay between the two groups (TSA, 11.3 days vs. DSA, 13.0 days; $\mathrm{P}=0.246)$. Postoperative complications included anastomotic leak, prolonged ileus, bleeding, wound infection, and pelvic collection.

Conclusion: The triple-staple technique is a safe alternative to double-staple anastomosis after anterior resection and effectively shortens operating time.

\section{Keywords: Stapling; Rectal anastomosis; Anastomotic leak; Anterior resection}

\section{INTRODUCTION}

A sound surgical technique is integral to optimizing postoperative outcomes and minimizing morbidity in colorectal surgery, especially anastomotic leakage. The reported clinical leakage rate after anterior resection varies from $3 \%$ to $20 \%$ [1-3]. Reports of the resulting morbidity and mortality vary considerably [1-3]. Many

Received: Oct 10, 2018 - Revised: Jun 14, 2019 - Accepted: Jun 30, 2019 Correspondence to: Christopher J Young, M.S., FRACS, FACS, FASCRS

RPAH Medical Centre, Suite 415/100 Carillon Avenue, Newtown, NSW 2042, Australia

Tel: +61-295290064, Fax: +61-1300078746

E-mail: cyoungnsw@aol.com

ORCID: https://orcid.org/0000-0002-7213-5137

\section{(C) 2021 The Korean Society of Coloproctology}

This is an open-access article distributed under the terms of the Creative Commons Attribution NonCommercial License (https://creativecommons.org/licenses/by-nc/4.0) which permits unrestricted noncommercial use, distribution, and reproduction in any medium, provided the original work is properly cited. risk factors are associated with anastomotic leakage and are categorized as patient-specific, intra-operative, and specific to low rectal anastomosis. The latter is attributed to the theory that low rectal resection with primary anastomosis poses more risks due to poor colonic vascularity and reduced remaining tissue available to support the anastomotic site. Patient-specific risk factors comprise malnutrition, steroids, tobacco use, leukocytosis, cardiovascular disease, alcohol use, and American Society of Anesthesiologists (ASA) score [4]. Other suggested factors that influence leakage rate are related to technique used and surgical expertise of the operator [1-4].

Surgeons have long sought to refine the technical aspects of constructing anastomoses with the goal of lowering the incidence of anastomotic leak. Introduction of surgical staplers has simplified constructing anastomoses, and this has contributed to their widespread clinical use, especially in the lower one-third of the rectum. Creation of anastomoses can still be difficult, especially in cases 
with poor exposure, such as in obese patients, male patients with a narrow pelvis, or patients with distal rectal tumors.

Various modifications in application of stapling techniques have evolved since their inception. The double-staple technique is now the most widely performed procedure and facilitates colorectal anastomoses at a lower level, minimizing the potential risk of fecal contamination $[5,6]$. The triple-staple technique utilizing stapled closure of both the proximal colon and distal rectal stump holds potential for a more rapid and secure colorectal anastomosis.

The aims of this study were to determine the safety and efficacy of the triple-staple technique and to compare the clinical outcomes with a historical control group for which the conventional double-staple technique had been performed.

\section{METHODS}

During a 26-month period from December 2015 to February 2018, 50 consecutive patients underwent rectal resection with triple-staple anastomosis (TSA) performed by a single surgeon. This cohort was compared to a consecutive group of 50 patients who underwent rectal resection with double-staple anastomosis (DSA) from June 2011 to November 2015, immediately prior to the TSA group, and performed by the same surgeon. Patients with locoregionally advanced rectal cancer or those with distant metastases who were not downstaged after neoadjuvant therapy precluding curative resection and subsequent anastomosis were not included in the study. Similarly, patients with low rectal tumors less than 5 $\mathrm{cm}$ from the anal verge and those involving the anal sphincter or requiring an emergency operation were also excluded.

The impetus to commence TSA was a case involving a 56-yearold female with cirrhosis, portal hypertension, and excessive bleeding, in which the $2 / 0$ prolene proximal purse-string for DSA could not be inserted due to the associated hemorrhage. The anastomosis was successfully completed with a TSA technique as described below, and the surgeon permanently changed his technique from DSA to TSA.

Data were collected for age, sex, ASA score, indication for surgery, mean operating time, mean length of postoperative hospital stay, and 30-day morbidity and mortality rates. Ethical approval of this study (X18-0344) was obtained from the Sydney South West Area Health Service Ethics Review Committee (RPAH Zone). Waiver of consent was granted by the Human Research Ethics Committee (HREC). The research involved no more than minimal risk to subjects, and the waiver or alteration did not adversely affect the rights and welfare of the subjects.

\section{Common to commencement of both techniques}

Following adequate mobilization of the rectum and left colon, the mesorectum, where appropriate, was divided below the anticipated lower margin of resection. A linear stapler was placed across the rectum at the distal margin of resection. A long rightangle clamp was placed proximal to the staple line (unless a sta- pling device was used to apply two separate linear staple lines and divides between) to prevent fecal soiling, and the rectum was divided along the edge of the staple line.

\section{Double-staple anastomosis technique}

A 2/0 prolene hand-sewn "whip-stitch" purse-string suture was placed at the proximal colon resection margin. The anvil shaft assembly was placed in the proximal bowel through the pursestring and was tied into the groove on the shaft.

\section{Triple-staple anastomosis technique}

The proximal colon resection margin was chosen and divided proximal to one hard, straight bowel clamp. The anvil of the circular stapler with an extension spike was placed into the proximal colon bowel lumen (Fig. 1). Control of the position of the spike inside the proximal bowel, usually with the left-hand, ensured no early or too proximal penetration of the colon bowel wall with the spike. A linear stapler was used to close the end of the proximal segment of the colon (Fig. 2), and the spike attached to the shaft of the anvil was then used to pierce the colon wall, just to either side of the linear staple line; the anvil head was then brought down flush with the linear staple line (Figs. 3, 4). The spike of the anvil shaft was then removed (Fig. 5).

\section{Common to completion of both techniques}

The circular stapler was introduced into the rectal segment with the anvil shaft assembly removed and the center rod retracted within the cartridge and was advanced through to either the ano-

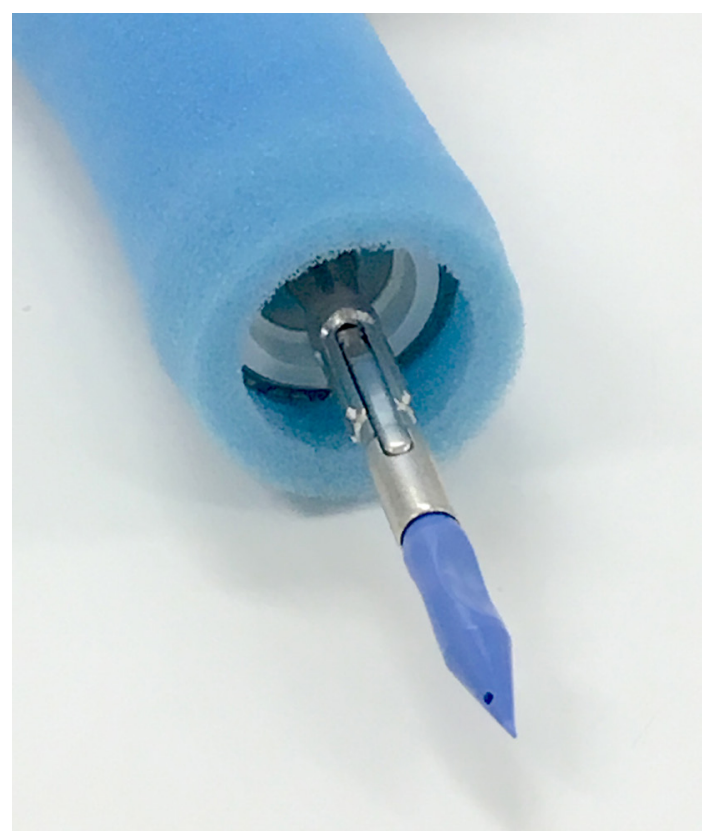

Fig. 1. An anvil of a circular stapler with an extension spike attached is inserted into the bowel lumen. 


\section{Annals of
Coloproctolog-Staple Technique Effective}

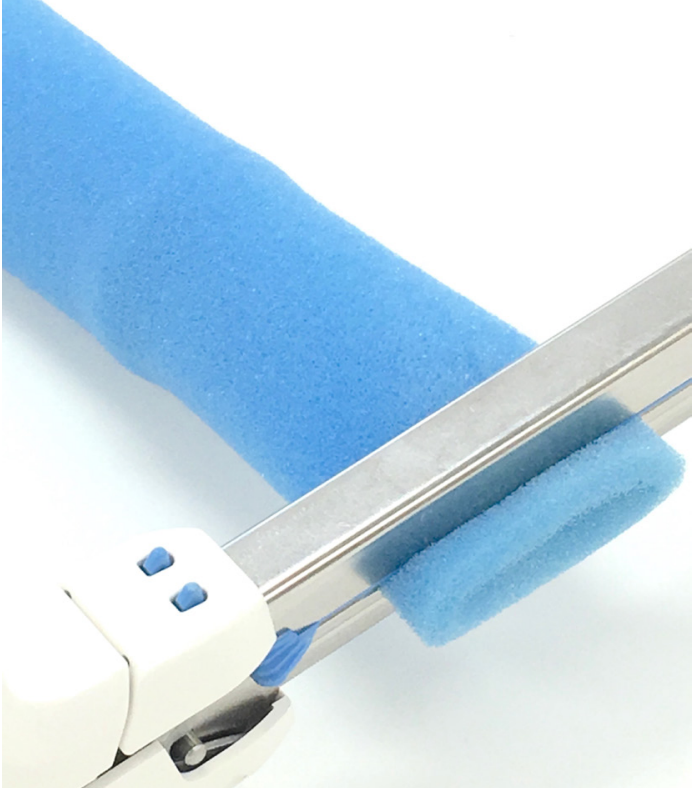

Fig. 2. A linear stapler is used to close the end of the proximal segment of the colon.

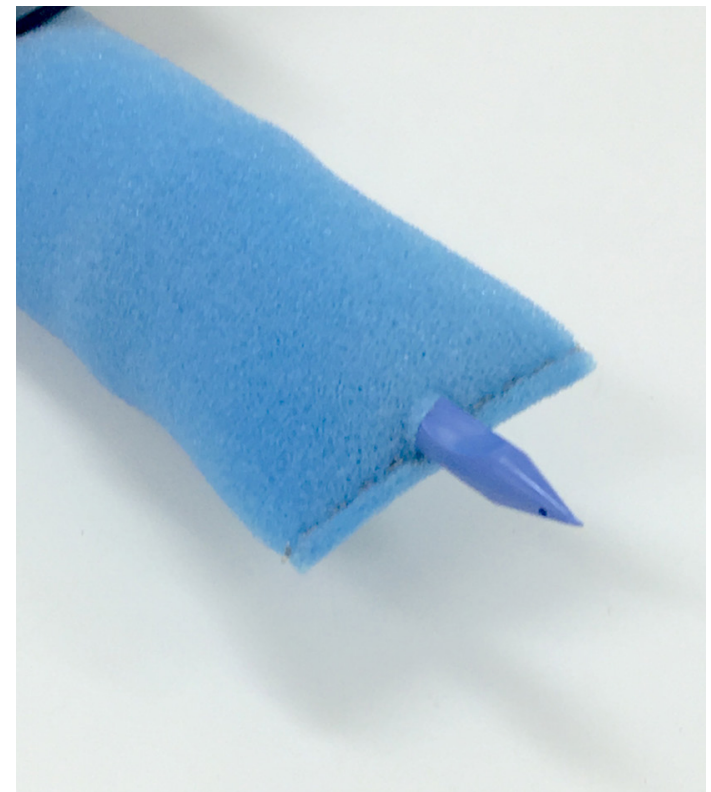

Fig. 3. The spike of the anvil is used to pierce the colon wall to just either side of the linear stapler line.

rectal junction or the rectal wall adjacent to the distal linear staple line. The anvil shaft was then inserted into the rod, and a circular end-to-end inverting anastomosis was completed.

All stapled anastomoses were tested for complete anastomotic doughnuts. Additionally, all anastomoses were both endoscopically inspected and tested for integrity by insufflation of air. This

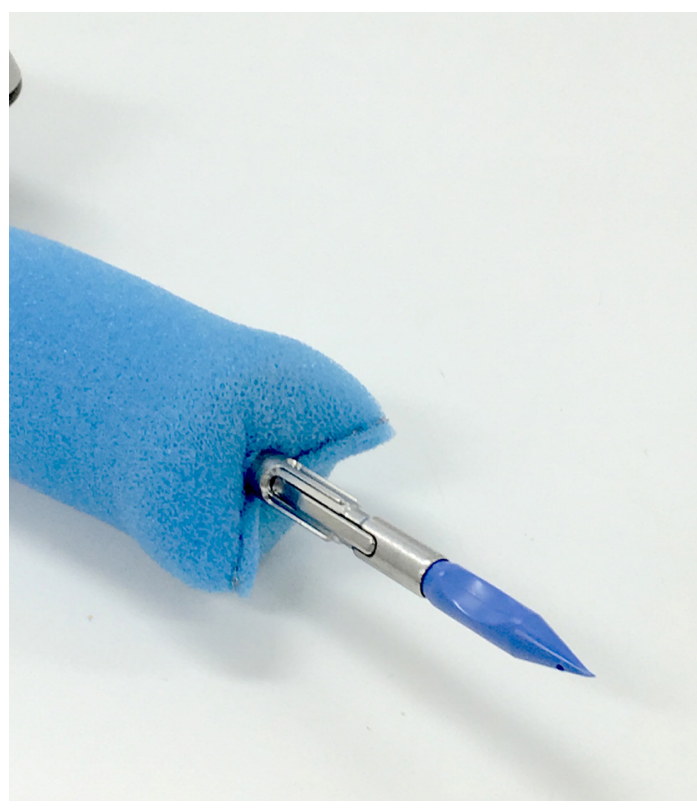

Fig. 4. The shaft of the anvil is brought down to be flush with the proximal end of the colon.

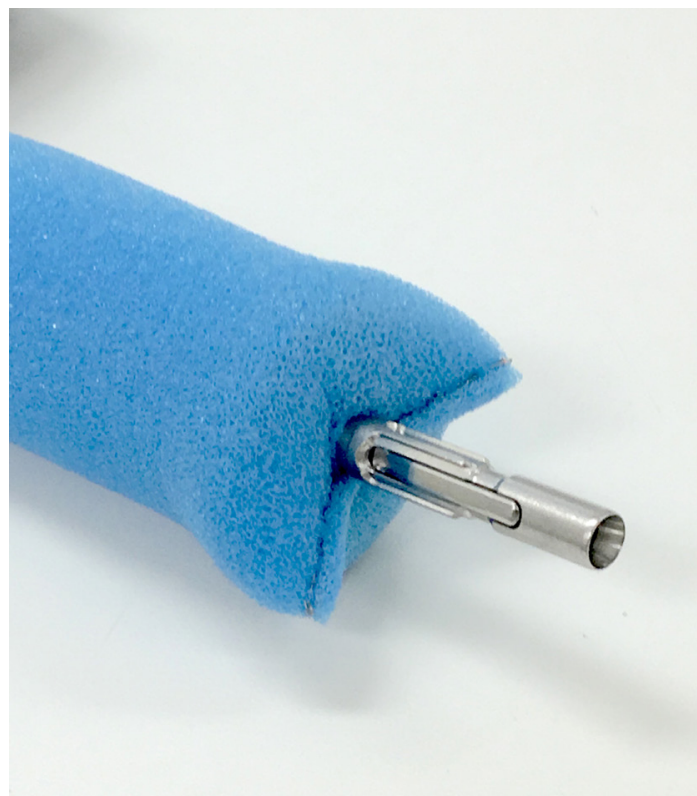

Fig. 5. The spike of the anvil shaft is removed prior to stapled anastomosis.

was achieved by occluding the proximal colon with a soft clamp across above the anastomosis while insufflating air to distend the colon. Leakage was detected by rising air bubbles.

Statistical analysis was completed using SPSS ver. 23.0 (IBM Corp., Armonk, New York, USA). Categorical variables were compared using the chi-square test, and continuous variables 
Table 1. Demographics and details of the operation

\begin{tabular}{lccc}
\hline Variable & DSA $(n=50)$ & TSA $(n=50)$ & P-value \\
\hline Age $(\mathrm{yr})$ & $65.8(38-87)$ & $62.2(31-82)$ & 0.098 \\
Sex & & & 0.009 \\
$\quad$ Male & $34(68.0)$ & $21(42.0)$ & \\
Female & $16(32.0)$ & $29(58.0)$ & \\
ASA score & & & 0.546 \\
1 & $16(32.0)$ & $16(32.0)$ & \\
2 & $27(54.0)$ & $23(46.0)$ & \\
3 & $7(14.0)$ & $11(22.0)$ & \\
Indication for surgery & & & 0.621 \\
Rectal cancer & $29(58.0)$ & $25(50.0)$ & \\
Diverticular disease & $11(22.0)$ & $13(26.0)$ & \\
Distal sigmoid cancer & $10(20.0)$ & $9(2.0)$ & \\
Other & $0(0)$ & $3(6.0)$ & \\
Covering ileostomy & $34(68.0)$ & $28(56.0)$ & 0.621 \\
Complication rate & $25(50.0)$ & $20(40.0)$ & 0.315 \\
Mean operating time (min) & 306.1 & 242.8 & 0.001 \\
Mean length of hospital stay (day) & 13 & 11.3 & 0.246 \\
\hline
\end{tabular}

Values are presented as mean (range) or number (\%).

VDSA, double-staple anastomosis; TSA, triple-staple anastomosis.

were compared using an independent-samples t-test. A P-value of $<0.050$ was considered statistically significant.

\section{RESULTS}

Demographic details of the patients are shown in Table 1. The mean age of the TSA group was 62.2 years (range, 31 to 82 years), compared with 65.8 years (range, 38 to 87 years) for the DSA group $(\mathrm{P}=0.098)$. Most of the patients in the TSA group were female $(58.0 \%)$, compared with the DSA group that was $68.0 \%$ male $(\mathrm{P}=0.015)$.

Most patients in both groups were ASA 2 with mild systemic disease $(\mathrm{P}=0.546)$. The most common indication for surgery in both groups was rectal cancer, followed by diverticular disease and distal sigmoid cancer $(\mathrm{P}=0.621)$. Three patients in the TSA group also had ischemic bowel, sigmoid volvulus, and appendiceal cancer with peritoneal metastases, respectively. There was no significant difference in number of covering loop ileostomies in the two groups (TSA, $56.0 \%$ vs. DSA, $68.0 \% ; \mathrm{P}=0.621$ ).

Mean operating time for the TSA group was significantly shorter than that for the DSA group (TSA, 242.8 minutes vs. DSA, 306.1 minutes; $\mathrm{P}=0.001$ ). There was no significant difference in complication rate (TSA, $40.0 \%$ vs. DSA, $50.0 \% ; \mathrm{P}=0.315$ ) or length of hospital stay between the two groups (TSA, 11.3 days vs. DSA, 13.0 days; $\mathrm{P}=0.246$ ).

Postoperative complications are outlined in Table 2. There were
Table 2. Comparison of postoperative complications between DSA and TSA groups

\begin{tabular}{lccc}
\hline Complication & DSA (\%) & TSA (\%) & P-value \\
\hline Anastomotic leak & $3(6.0)$ & $1(2.0)$ & 0.307 \\
Ileus/obstruction & $11(22.0)$ & $5(10.0)$ & 0.086 \\
High stoma output & $3(6.0)$ & $3(6.0)$ & 1.000 \\
Urinary tract infection & $4(8.0)$ & $1(2.0)$ & 0.169 \\
Pelvic collection & $3(6.0)$ & $1(2.0)$ & 0.307 \\
Hemorrhage & $2(4.0)$ & $2(4.0)$ & 1.000 \\
Wound infection & $4(8.0)$ & $3(6.0)$ & 0.654 \\
Pneumonia & $2(4.0)$ & $3(6.0)$ & 0.646 \\
Arrhythmia & $0(0)$ & $3(6.0)$ & 0.079 \\
\hline
\end{tabular}

DSA, double-staple anastomosis; TSA, triple-staple anastomosis.

no deaths or major cardiopulmonary complications in either group. One clinical anastomotic leak $(2.0 \%)$ was recorded in the TSA group and $3(6.0 \%)$ in the DSA group $(\mathrm{P}=0.307)$. All three patients in the DSA group had a covering ileostomy at initial resection; 2 were managed with percutaneous drainage and antibiotics and 1 with antibiotics alone. The patient in the TSA group with a clinical leak was a 65 -year-old male with a body mass index of 52, who did not have a covering ileostomy. He required laparotomy, washout, and proximal diversion. There were no radiologic leaks in either group. Other postoperative complications including hemorrhage from the anastomotic staple line, prolonged ileus, obstruction, wound infection, and respiratory complications were similar in the groups.

\section{DISCUSSION}

Anastomotic leak is the most feared colorectal resection complication and is the primary outcome by which success of rectal anastomosis is measured. There is concern that use of more intersecting staple lines on the anastomoses may increase the risk of anastomotic leak. Although this concern might seem justified, Julian and Ravitch [7] addressed this issue in experimental studies with dogs, showing that linear staples are usually removed with doughnuts and are deformed, cut, or squeezed out. Another experimental study further demonstrated that intersecting staple lines in small bowel and colonic anastomoses do not reduce anastomotic blood flow to a level that may potentially increase the risk for anastomotic leakage [8]. Reports of increasing clinical experience also attest to the safety of stapling across a staple line $[2,3]$.

The double-staple technique is currently the most widely performed technique in colorectal anastomoses $[3,5,9]$. It has been associated with shorter operation time, minimal contamination, a lower rate of covering colostomy, and the ability to create a very low anastomosis compared to a hand-sewn technique. This translates to sphincter-saving surgery and permanent stoma avoidance. Double-staple anastomoses, however, have been associated with 
anastomotic stenosis varying between $3 \%$ and $21 \%$ and risk of pelvic sepsis due to fecal contamination $[3,5,9]$. Use of a pursestring suture on the proximal colon poses a contamination risk upon opening of the bowel lumen. Moreover, the difficulty associated with purse-string placement in patients with dilated bowel due to obstruction is a commonly encountered concern. Size disparity between the 2 ends of the bowel in such obstructed patients results in technical difficulty in purse-string placement and may fail to include the entire circumference of the bowel wall regardless of whether manual or automatic purse-string devices are used.

Illuminati et al. [5] reported his results using DSA in 108 patients with carcinoma of the rectum and found a leakage rate of $6 \%$. Meanwhile, Redmond et al. [10] reported a leakage rate of $9 \%$ and perioperative mortality of $2 \%$ in 111 patients who underwent rectal resection with DSA. Both studies concluded that DSA allowed safe low anterior rectal resection, with low mortality and anastomotic leakage rates. Similarly, Mahid et al. [11] reported the results of his review of 291 consecutive patients who underwent rectal resection with TSA and noted a low anastomotic leakage rate of $1 \%$. The anastomotic leakage rate in this study of $50 \mathrm{pa}-$ tients in the DSA group was $6.0 \%$, which is comparable to rates in the literature. While the anastomotic leak rate in the TSA group was lower $(2.0 \%)$, this result was not significantly different from that of the DSA group.

In patients with low rectal anastomosis, TSA may be appropriate and result in better postoperative outcomes compared to existing stapling methods such as the DSA. Elimination of purse-string placement in both the proximal and distal bowel stumps using the triple-staple technique addresses size disparity concerns between the two ends of the bowel in obstructed patients. It does not eliminate the need to open the proximal bowel but can help minimize fecal spillage. The additional cost for a reload cartridge of the linear stapler may be justified by the operating room time saved and the low rate of anastomotic leak using this technique [11].

There are limitations to the conclusions that can be drawn from this study because of the number of patients and the historical control design. This study demonstrated that TSA is associated with a shorter mean operating time compared to DSA. The additional operating time in the DSA group may be attributed to the time required to construct a purse-string or correct a leak. However, this may also be explained by the male predominance of patients in the DSA group, whose narrower pelvis that can result in more difficult and time-consuming dissection. In conclusions, the triple-staple technique is a safe alternative to double-staple anas- tomosis after anterior rectal resection and effectively shortens operating time.

\section{CONFLICT OF INTEREST}

No potential conflict of interest relevant to this article was reported.

\section{REFERENCES}

1. Neutzling CB, Lustosa SA, Proenca IM, da Silva EM, Matos D. Stapled versus handsewn methods for colorectal anastomosis surgery. Cochrane Database Syst Rev 2012;(2):CD003144.

2. Balaji NS, Macklin CP, Fawole S, Aster AS, Rao VS, Moore PJ, et al. The 'modified triple staple' technique: a variant stapling technique for anastomosis after low anterior resection. Surgeon 2007; 5:199-201.

3. Slieker JC, Daams F, Mulder IM, Jeekel J, Lange JF. Systematic review of the technique of colorectal anastomosis. JAMA Surg 2013;148:190-201.

4. Kingham TP, Pachter HL. Colonic anastomotic leak: risk factors, diagnosis, and treatment. J Am Coll Surg 2009;208:269-78.

5. Illuminati G, Carboni F, Ceccanei G, Pacile MA, Pizzardi G, Palumbo $P$, et al. Long-term evaluation of a modified double staple technique for low anterior resection. Acta Chir Belg 2014;114: 338-43.

6. Hunt LE, Christmas B, Galandiuk S. Elimination of the pursestring suture facilitates stapled colorectal anastomosis/triple staple technique for low rectal anastomosis. Gastroenterology 2000; 118:A1517.

7. Julian TB, Ravitch MM. Evaluation of the safety of end-to-end (EEA) stapling anastomoses across linear stapled closures. Surg Clin North Am 1984;64:567-77.

8. Zilling T, Walther BS. Are intersecting staple lines a hazard in intestinal anastomosis? Dis Colon Rectum 1992;35:892-6.

9. Radovanovic Z, Petrovic T, Radovanovic D, Breberina M, Golubovic A, Lukic D. Single versus double stapling anastomotic technique in rectal cancer surgery. Surg Today 2014;44:1026-31.

10. Redmond HP, Austin OM, Clery AP, Deasy JM. Safety of doublestapled anastomosis in low anterior resection. Br J Surg 1993;80: 924-7.

11. Mahid S, Galandiuk S, Christmas B, Tran D. Triple-staple technique for low rectal anastomoses eliminates the purse-string suture and facilitates stapled colorectal anastomosis. J Am Coll Surg 2006;202:382-3. 